\title{
Adenocarcinoma mucinoso pulmonar con patrón de células en anillo de sello. Una entidad infrecuente
}

\author{
FRANCISCO JAVIER TORRES G.*, PILAR FERNÁNDEZ M.*, \\ DOMINGO JIMÉNEZ G.* y JULIO MARQUÉS A.*
}

\section{Mucinous adenocarcinoma of the lung with signet-ring cell pattern. An infrequent entity}

Mucinous adenocarcinoma of the lung with signet-ring cell pattern is an infrequent entity of easy diagnosis. We report the case of a 55 year-old patient with preexisting ischemic heart disease and recent diagnosis of pulmonary adenocarcinoma with signet-ring cell pattern. It is necessary discard a primary extrapulmonar location (digestive tract). Immunohistochemistry is a useful tool in the differential diagnosis.

Key words: Mucinous adenocarcinoma, lung, signet-ring cells.

\section{Resumen}

El adenocarcinoma mucinoso de pulmón con patrón de células en anillo de sello es una entidad infrecuente de diagnóstico fácil. Presentamos el caso de un paciente de 55 años con antecedente de cardiopatía isquémica y diagnóstico actual de adenocarcinoma primario pulmonar con patrón de células en anillo de sello. Es necesario descartar un origen primario extrapulmonar, principalmente de localización digestiva. Las técnicas inmunohistoquímicas son de gran utilidad a la hora de establecer diagnósticos diferenciales.

Palabras clave: Adenocarcinoma mucinoso, pulmón, células en anillo de sello.

\section{Introducción}

El adenocarcinoma con patrón de células en anillo de sello primario de pulmón es una neoplasia infrecuente con entidad propia, encuadrada dentro de los adenocarcinomas mucinosos de pulmón. Sus características morfológicas son muy singulares si bien es necesario descartar un origen primario extrapulmonar, sobre todo a nivel de tracto digestivo, mama y próstata. El diagnóstico diferencial de las lesiones primarias de pulmón se establece principalmente con otras lesiones mucinosas.

\section{Caso clínico}

Paciente varón de 55 años, no fumador, jubilado debido a cardiopatía isquémica (IAM, HTA e hipercolesterolemia) que acude a consulta de Neumología debido a tos seca de tres meses de evolución que se ha hecho productiva. El paciente no refiere disnea, dolor torácico, fiebre ni síndrome constitucional. La exploración física no demuestra sibilancias aunque sí hipoventilación marcada en pulmón derecho, con presencia de sibilantes, algunos inspiratorios en la base, acompañados de crepitantes. Se realiza un estudio radiológico de tórax siendo la radiografia anteroposterior patológica (pérdida de volumen de hemitórax derecho, leve patrón intersticial homolateral y aumento de la densidad paratraqueal derecha) (Figura 1A). Dichos hallazgos justifican la realización de TAC torácica observándose la presencia de una tumoración pulmonar derecha acompañada de adenopatías hiliares, subcarinales y mediastínicas así como un engrosamiento del manguito peribronco-vascular a dicho nivel

* Unidad de Anatomía Patológica, Unidad de Neumología, Unidad de Diagnóstico por Imagen. Hospital de Alta Resolución de Utrera. Sevilla. España. 
(Figura 1B, 1C, 1D); la imagen de linfangitis carcinomatosa era evidente. Del mismo modo se realiza un estudio endoscópico del árbol bronquial, tomándose biopsias y muestras citológicas de cepillado y lavado-aspirado bronquioalveolar, que son remitidas al laboratorio de Anatomía Patológica.

El estudio citológico de las muestras correspondientes al cepillado bronquial resultó negativo para células neoplásicas; en cambio, al analizar las muestras de aspirado bronquial y lavado bronquioalveolar, llamaba la atención la presencia de células vacuoladas con desplazamiento e incluso indentación del núcleo por la vacuola (Figura 2A). Dichas células mostraban gran parecido a células glandulares procedentes de la descamación del epitelio respiratorio por lo que se esperó a la lectura de las biopsias para realizar una adecuada correlación entre los hallazgos.

Se recibieron en el laboratorio de Anatomía Patológica varios fragmentos irregulares, blanquecinos y milimétricos que se procesaron según rutina. El estudio histológico demostró la presencia de un epitelio bronquial sin alteraciones histológicas significativas; en cambio, a nivel de la lámina propia se pudieron observar múltiples grupos de células mucinosas en el seno de lagos mucosos bien definidos. Muchas de estas células mucinosas mostraban una morfología en anillo de sello similar a la exhibida por las células identificadas en citología (Figura 2B, 2C). La aplicación de técnicas histoquímicas tales como el PAS-Azul alcián pusieron de manifiesto los lagos mucinosos y el contenido de las vacuolas mucinosas (Figura 2D). El carácter infiltrativo de la lesión así como las características celulares permitieron realizar el diagnóstico de adenocarcinoma mucinoso con patrón de células en anillo de sello. Quedaba por definir el verdadero origen de la neoplasia. Se recomendó la realización de estudios endoscópicos del tubo digestivo para descartar un origen primario en esta localización (recordemos que el estómago y el colon son las localizaciones más frecuentes de este tipo de tumores sin contar la mama, en mujeres). La negatividad de dicho estudio permitió plantear la posibilidad de que se tratase de un adenocarcinoma primario de pulmón. A ello contribuyeron asimismo los estudios inmunohistoquímicos realizados sobre el escaso material disponible (CK7 y CK20 negativo). No se realizaron tinciones para TTF1 y CDX2 por falta de disponibilidad de dichos marcadores. La evolución del cuadro fue fulminante y el paciente falleció dos meses después del diagnóstico, antes de que se pudieran realizar más estudios.

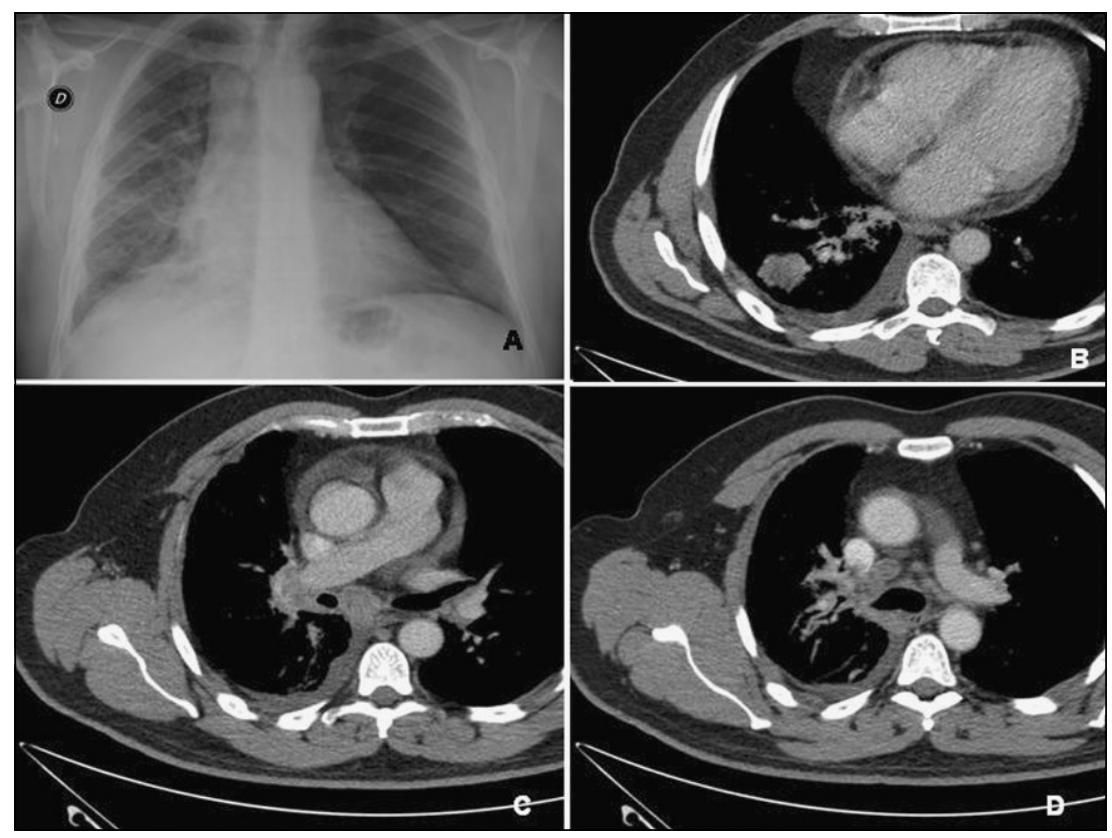

Figura 1. Adenocarcinoma mucinoso de pulmón con patrón de células en anillo de sello. A: Radiografía anteroposterior de tórax. B: TAC. Tumoración. C: TAC. Adenopatía hiliar. D: TAC. Adenopatía peritraqueal y subcarinal (ver texto). 


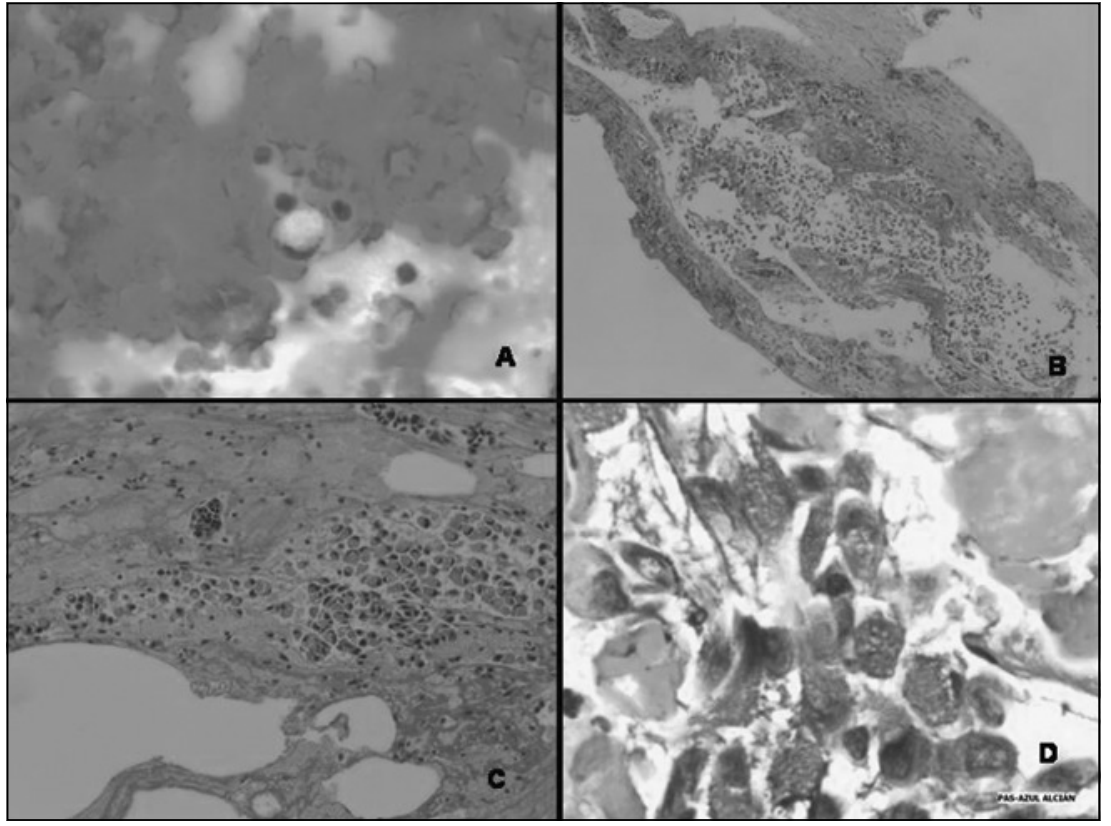

Figura 2. Adenocarcinoma mucinoso de pulmón con patrón de células en anillo de sello. A: Aspirado bronquial: células vacuoladas. PAP. 100x. B: Infiltración neoplásica parenquimatosa. HE 200x. C: Histología. Detalle celular. HE. 200x. D: Detalle citológico. PAS-Azul alcián. 400x (ver texto)

\section{Discusión}

El adenocarcinoma con patrón de células en anillo de sello es una entidad sumamente infrecuente $(0,14-1,9 \%$ de los carcinomas de pulmón) e independiente, recogida como tal en la clasificación de tumores pulmonares de la OMS de 2004 Se trata de un subtipo de adenocarcinoma productor de mucina caracterizado por la presencia de células redondas que poseen una gran vacuola citoplasmática que desplaza el núcleo a la periferia, adoptando ese fenotipo tan característico que tradicionalmente se ha comparado con el de un anillo de sello.

El patrón de células en anillo de sello se corresponde con un comportamiento agresivo de la neoplasia, habiéndose definido una serie de datos con cierta capacidad pronóstica. Nos referimos a la edad del/ la paciente, al porcentaje de células en anillo de sello identificadas sobre el total de células o estructuras neoplásicas y a la invasión vascular y linfática. Porcentajes mayores del 50\% se suelen observar en edades más tempranas y se asocian a una mayor probabilidad de diseminación metastásica $(28 \%$ de supervivencia a los 5 años frente a un $50 \%$ en aquellos casos con porcentajes inferiores al $50 \%)^{2-3}$.

Como hemos comentado al describir el caso clínico, y debido a la infrecuencia de este tipo de neoplasias a nivel pulmonar, es requisito imprescindible descartar una localización primaria extrapulmonar, principalmente en tubo digestivo (estómago y colon), próstata y mama, localizaciones en las que el mencionado fenotipo es más frecuente. En este sentido, además de a la clínica, es útil recurrir a las técnicas inmunohistoquímicas; las neoplasia primarias pulmonares suelen ser positivas para el TTF-1 y negativas para el CDX2 (lo contrario ocurre con las neoplasia digestivas). También es útil analizar el perfil de citoqueratinas CK7 y CK20. Mientras que las neoplasias primarias de pulmón suelen ser positivas para CK7 y negativas para CK 20, las neoplasias de colon suelen ser positivas para CK20 y negativas para CK7. La negatividad de las neoplasias de pulmón y tracto digestivo para PSA facilita el diagnóstico diferencial con respecto a los tumores prostáticos $^{4-5}$.

Una vez realizados los estudios pertinentes podemos comenzar a enfocar la neoplasia como primaria de pulmón. No obstante, será necesario llevar a cabo una serie de diagnósticos diferenciales para los cuales cobra gran importancia obtener los datos clínicos del/la paciente. Si nos ceñimos a la clasificación de tumores pulmonares de la OMS 2004르 este diagnóstico diferencial deberá ser establecido fundamentalmente con otras neoplasias mucinosas.

El carcinoma coloide pulmonar es una neoplasia que exhibe las mismas características que en otras localizaciones en las que es más frecuente. Se caracteriza por extensos lagos de mucina en cuyo seno pueden observarse nidos de células epiteliales neoplásicas con variable atipia. Estas 
células no tienen por qué tener morfología en anillo de sello. Realmente se trata de una variante de adenocarcinoma mucinoso fácil de identificar e interpretar. Distinto es el caso del adenocarcinoma de células globoides, hoy incluido e identificado con el adenocarcinoma bronquioloalveolar de tipo mucinoso. Es cierto que pueden existir ciertas analogías en cuanto a las características celulares pero, fijémonos bien en las mismas; mientras que en las células en anillo de sello la gran vacuola citoplasmática desplaza al núcleo a la periferia, en el caso del carcinoma bronquioloalveolar la célula protagonista es columnar y el desplazamiento nuclear se realiza hacia la base celular. Por otro lado, mientras que es frecuente observar pleomorfismo nuclear en el fenotipo de anillo de sello, la variante mucinosa de carcinoma bronquioloalveolar es considerada de bajo grado nuclear. Ya hemos propuesto un criterio de distinción: el núcleo. Si persisten las dudas, recurriremos a la clínica pues el carcinoma bronquioloalveolar, al mostrar un crecimiento septal (tabiques alveolares) originará un cuadro clínico y radiológico similar al de una neumonía, focal, lobar o total de acuerdo con la extensión de la neoplasia ${ }^{6,7}$.

El diagnóstico diferencial puede asimismo ser extensivo a las neoplasias sólidas con componente mucinoso, pero en este caso el componente sólido será el que guíe nuestro diagnóstico.

Del mismo modo podemos estar frente a una neoplasia mixta en la que es necesario describir cada uno de los componentes observados y, a ser posible, su porcentaje. Esta tarea se ve facilitada en piezas quirúrgicas de gran tamaño en las que podemos realizar seriaciones parenquimatosas. En nuestro caso sólo pudimos tener acceso a una pequeña biopsia endoscópica. No obstante, el material fue suficiente para realizar el diagnóstico. El comportamiento de nuestra neoplasia fue muy agresivo. Se detectó una "mancha" hepática que no pudo ser estudiada y el paciente murió dos meses después de haber sido diagnosticado.

Distintos estudios han intentado caracterizar histoquímicamente e inmunohistoquímicamente el adenocarcinoma con patrón de células en anillo de sello pulmonar. Moon y cols ${ }^{3}$, intentando establecer semejanzas y diferencias respecto al carcinoma difuso pulmonar, observaron que existía ausencia o localizaciones aberrantes en la expresión de E-caderina y beta-catenina al contrario que lo que ocurre en estómago y colon. Por su parte, Hayashi y cols ${ }^{5}$, demostraron positividad para MUC 1 y negatividad para MUC2 mientras que la localización colónica mostraba un patrón inverso.
Tal como se deduce de las cifras de supervivencia, el patrón de células en anillo de sello conlleva un pronóstico ominoso que se deriva del carácter infiltrativo lesional. No siempre será posible realizar cirugía, recurriéndose en muchos casos a la quimioterapia, generalmente paliativa y poco efectiva ${ }^{4}$.

\section{Bibliografía}

1.- COLBY $\mathrm{T} \mathrm{V}$, NOGUCHI $\mathrm{M}$, HENSCHKE $\mathrm{C}$, VAZQUEZ M F, GEISINGER K, YOKOSE T, et al. Adenocarcinoma. In Pathology and genetics: Tumors of the lung, pleura, tymus and heart. Travis WD, Brambilla E, Muller-Hermelink HK, Harris CC (editors). IARC: Lyon, France 2004, pág. 35.

2.- $\quad$ TSUTA K, ISHII G, YOH K, NITADORI J, HASEBE T. NISHIWAKI Y, et al. Primary lung carcinoma with signet-ring cell components: clinicopathological analysis of 39 cases. Am J Surg Pathol 2004; 28: 868-74.

3.- MOON K C, CHO S Y, LEE H S, JEON Y K, CHUNG $\mathrm{J}$ H, JUNG K C, et al. Distinct expression patterns of Ecadherin and beta-catenin in signet-ring cell carcinoma components of primary pulmonary adenocarcinoma. Arch Pathol Lab Med 2006; 130: 1320-25.

4.- HIRAKI A, UEOKA H, YOSHINO T, TABATA M, KIURA K, TANIMOTO Y, et al. Primary signet-ring cell carcinoma of the lung with histochemical characterization. Anticancer Res 2002; 22: 1079-81.

5.- HAYASHI H, KITAMURA H, NAKATANI Y, INAYAMA Y, ITO T. Primary signet-ring cell carcinoma of the lung: histochemical and immunohistochemical characterization. Hum Pathol 1999; 30: 378-83.

6.- TSUTA K, SHIBUKI Y, MAEZAWA N, TOCHIGI N, MAESHIMA A M, SASAJIMA Y, et al. Cytological features of signet-ring cell carcinoma of the lung: comparison with the globet cell type adenocarcinoma of the lung. Diagnostic Cytopathology 2009; 37: 159-63.

7.- TSUTA K, ISHII G, NITADORI J, MURATA Y, KODAMA T, NAGAI K, et al. Comparison of the immunophenotypes of signet-ring cell carcinoma, solid adenocarcinoma with mucin production and mucinous bronchioloalveolar carcinoma of the lung characterized by the presence of cytoplasmic mucin. J Pathol 2006; 209: 78-87.

Correspondencia a:

Dr. Francisco Javier Torres Gómez

Hosp. De Alta Resolución de Utrera

Dirección: Avda. Brigadas Internacionales S/N

41710. Utrera. Sevilla, España.

E-mail: javiertorresgomez@yahoo.es 